0 Maiga, B Reime, A Hahn, B Kreuels, W Loag, J May. Infectious Disease Epidemiology, Bernhard-Nocht-Institute for Tropical Medicine, Hamburg, Germany

Background and Aims In Ghana, 74/1,000 children per year die before their fifth birthday and many suffer from ill health. We explore the relative impact of genetic traits for the children's health. Methods Within a trial on Intermittent Preventive Treatment in Infants for malaria 1,070 infants were recruited at 3 months and followed-up for 21 months in the Ashanti region. Each month standardized medical history, clinical exam and parasite density were taken. Anthropometric measures were performed every 3 months. DNA preparation and genotyping were performed according to standardized protocols. We defined "health" as a lack of malaria episodes with high parasitaemia, no episodes of anemia, no reporting of severe events like measles, accidents, burns, pneumonia, and normal WHO-growth standards during the entire study phase. We tested the association between health and genetic traits in multivariable logistic regression analyses adjusted for socioeconomic, spatial and clinical data.

Results As expected, children with sickle cell trait (HbAS) were more likely to grow up healthy $(\mathrm{OR}=2.89,95 \%-\mathrm{CI}=1.59-5.24)$. This effect was less pronounced for carriers of $\mathrm{HbAC}$ (hemoglobin $\mathrm{C}$ ). The CD74 gene SNP rs7709772AG was associated with health $(\mathrm{OR}=8.00,95 \%-\mathrm{CI}=1.76-36.29)$. This gene encodes for a membrane protein which is important for the regulation of immune responses against infectious diseases. $\alpha$-thalassemia was not related to health in multivariate analyses.

Conclusions Independently of socioeconomic and geographic factors, genetic traits that influence the risks for malaria and other infectious diseases may affect children's health in an endemic area. This information can be relevant for the development of treatments.

\title{
1680 AN AUDIT OF IMPACTS OF FRENULECTOMY IN BREAST FEEDING
}

doi:10.1136/archdischild-2012-302724.1680

'E Matthews, ${ }^{2} \mathrm{M}$ Choudhry. ${ }^{1}$ Community Midwives, Imperial College Healthcare NHS Trust; 2Paediatric Surgery, Chelsea and Westminster Hospital, Imperial College, London, UK

Background and Aims To review impact of frenulectomy on breast feeding success.

Methods Prospective audit of babies undergoing frenulectomy from December 2010 to January 2012. A pre-procedure questionnaire was completed in all cases. Babies were assessed and procedure was carried out in the clinic under complete aseptic conditions. Babies were breastfed immediately after treatment and observed for 30 minutes. No further clinical review was conducted. A post-procedure questionnaire was returned within 3 weeks.

Results 50 babies underwent frenulectomy. Mean age at procedure was 6.6 weeks and range 1.1-22.3 weeks. Procedures were carried out by a single operator without complications. 26 post procedure questionnaires were returned. Pre and post feeding methods and difficulties are shown in the tables below.

Abstract 1680 Table 1 Breast feeding methods pre and post frenulectomy

\begin{tabular}{lcc}
\hline Feeding Method & Pre-frenulectomy - n [\%] & Post-frenulectomy - n [\%] \\
\hline Breast feeding only & $12[46 \%]$ & $16[61 \%]$ \\
Artificial milk only & $1[4 \%]$ & $2[8 \%]$ \\
Expressed breast milk only & $0[0 \%]$ & $0[0 \%]$ \\
Breast feeding combined with & $13[50 \%]$ & $8[31 \%]$ \\
expressed and/or artificial milk & & \\
\hline
\end{tabular}

Abstract 1680 Table 2 Feeding difficulties pre and post frenulectomy

\begin{tabular}{lcc}
\hline Feeding difficulties & Pre-frenulectomy n [\%] & Post-frenulectomy n [\%] \\
\hline None & $3[12 \%]$ & $14[54 \%]$ \\
Sore nipples/breast & $13[50 \%]$ & $5[19 \%]$ \\
Difficulty attaching/maintaining & $16[62 \%]$ & $2[8 \%]$ \\
Prolonged feeding/unsatisfied & $11[42 \%]$ & $9[35 \%]$ \\
Poor weight gain & $5[19 \%]$ & $1[4 \%]$ \\
\hline
\end{tabular}

Conclusions Frenulectomy has a positive impact on breastfeeding success. We recommend early treatment if feeding difficulties are observed combined with tongue tie.

\section{LATE-PRETERM DELIVERY: PSYCHOLOGICAL DISTRESS AND LACTOGENESIS}

doi:10.1136/archdischild-2012-302724.1681

C Gabrieli, R Ciullo, L Sansone, I Gambina, F Cavallin, D Faggian, M Santini, V Zanardo. Padua University School of Medicine, Agna, Italy

Late-preterm infants represent the most rapidly growing group of preterm births. The majority of these infants is treated as term neonates at the regular nursery.

It is noteworthy that late-preterm infants have a lower breastfeeding prevalence than term neonates.

It is not known whether psychological distress affects lactogenesis or the mother-to-infant bonding.

The study's aim is to verify if late-preterm delivery is associated to psychological distress affecting Mother-to-Infant bonding and breastfeeding.

The study was performed at the Departement of Gynaecology and Human. Reproduction Science of Padua University, between June and October 2011.

The study group consisted of 75 mothers: 25 delivered late-preterm with caesarean section and 50 delivered with term vaginal birth.

The third day after birth, four psychological tests were administered to mothers: STAI, EPDS, MIBS, LüscherColorTest.

Baseline levels of prolactin and cortisol were controlled.

ANOVA shows significantly higher levels of trait anxiety and state anxiety in late-preterm infants' mothers: the average trait anxiety is 46.0 (IOR 39.0-50.0) in late-preterm infants' mothers and 40.5 (IOR35.3-44.0) in term infants' mothers ( $\mathrm{p}=0.01$ ); and the average state anxiety is 48.0 (IOR42.0-56.0) in late-preterm infants' mothers and 43.0 (IOR 39.0-48.8) in term infants' mothers ( $p=0.03$ ) respectively. There are significantly higher levels of depression in late-preterm infants' mothers: in EPDS the average is 9 (IOR 8-13) in late-preterm infants' mothers and 6 (IOR 4-9) in term infants' mothers $(p=0.001)$. In MIBS, joy is lower in late-preterm infants' mothers.

In late preterm infants this is associated to reduced exclusive breastfeeding at discharge ( $20 \%$ vs $66 \%$ ).

The LüscherColourTest is comparable for both groups.

Mothers who delivered late-preterm have negative emotional changes, without hormonal changes. The psychological distress is negatively associated to an altered Mother-to-Infant bonding and lactation performance.

\section{NICE (UK) GUIDELINES FOR SEDATION IN CHILDREN: NEED FOR A PRAGMATIC APPROACH?}

doi:10.1136/archdischild-2012-302724.1682

G Erumbala, I Misra, N Chawda. Paediatrics, Milton Keynes General Hospital NHS Foundation Trust, Milton Keynes, UK 